\title{
Impact of High-normal Serum TSH with First Trimester Pregnancy Loss: A Case-control Study in Tertiary Care Hospitals in Bangladesh
}

\author{
Yisrat Jahan ${ }^{1, *}$, M Anwar Hussain ${ }^{2}$, Rezaul Karim Kazal ${ }^{2}$, \\ Mohammad Akhteruzzaman 3 , and Rifat Jahan ${ }^{4}$ \\ ${ }^{1}$ Maternal and Child Health Institute, Bangladesh \\ ${ }^{2}$ Department of Obstetrics and Gynaecology, Bangabandhu Sheikh Mujib Medical University \\ ${ }^{3}$ Socio-Economic Infrastructure Division, Ministry of Planning \\ ${ }^{4}$ Biomedical Research Foundation, Bangladesh \\ *Corresponding author: yisratjahan@gmail.com
}

Received: May 17, 2017; revised: December 29, 2017; accepted: January 12, 2018.

\begin{abstract}
The relationship between existing thyroid autoimmunity and the probability of spontaneous abortion has been the subject of a number of studies. In this study, we evaluate the impact of 'high normal' serum TSH levels (2.5-5.0 mlU/L) on first trimester pregnancy loss in anti-TPO Ab negative women in Bangladesh. Specifically, we wanted to know the average serum TSH levels for women who had miscarriage (case group) and who did not have miscarriage (control group) during the first trimester of pregnancy. Unmatched case control study involving 119 women in their first trimester was conducted during July 2011 to June 2012 in three tertiary care hospitals in Bangladesh. We found average TSH level for the control group $(1.42 \pm 0.86 \mathrm{mlU} / \mathrm{L})$ to be lower than the average for the case group $(1.74 \pm 1.15 \mathrm{mlU} / \mathrm{L})$. However, we did not find statistically significant association between the serum TSH level and first trimester pregnancy loss. The findings of this study will provide a baseline for future studies on this topic involving Bangladeshi women. In addition, our study adds to the growing discussion on redefining the lower limit for the 'high normal' serum TSH level among the anti-TPO Ab negative women.
\end{abstract}

Keywords: TSH level, anti-TPO Ab negative, pregnancy loss, first trimester 


\section{Introduction}

The thyroid hormones disorders are among the most common endocrine disorders found in pregnant women. A number of studies have shown that maternal thyroid hormone imbalance may influence the outcomes of mother and fetus at all stages of pregnancy (Casey and Leveno, 2006). Disorders due to hypothyroidism is the most common and has been associated with placental abruption, reduced intellectual function in the offspring, pregnancyinduced hypertension, preterm delivery, and fetal loss (De Groot et al., 2012). Measurement of serum thyroid stimulating hormone (TSH) and thyroid peroxidase antibodies (TPO-Ab) are two common ways to assess maternal thyroid status (Quinn et al., 2005). It has been reported that the presence of antibody to thyroid peroxidase is associated with increased risk of miscarriage, preterm birth and maternal postpartum thyroid disease (Stricker et al., 2007).

Although the exact level of TSH that is indicative of risk of pregnancy loss is not known, a number of studies have suggested a range of values for serum TSH level that are associated with first trimester pregnancy loss. For instance, a follow up study involving 343 Chinese women reported a normal range for first-trimester TSH levels of 0.03-2.3 mIU/L (Panesar et al., 2001). In a study of 585 thyroid antibody-negative women, Pearce et al. (2008) found $95 \%$ of TSH levels were between 0.04 and $3.6 \mathrm{mIU} / \mathrm{L}$. Study conducted in 1817 Australian women (Gilbert et al., 2008), who were between 9 and 13 weeks of gestation reported a normal TSH range of 0.02-2.15 mIU/L. Stricker et al. (2007) screened 783 thyroid antibody-negative women from Geneva, Switzerland, and reported a 95\% confidence interval for TSH level to be $0.08-2.83 \mathrm{mIU} / \mathrm{L}$. It has been suggested that presence of maternal thyroid autoantibody and high normal level of TSH (2.5 to $5.0 \mathrm{mIU} / \mathrm{L}$ ) is strongly associated with miscarriage (Ghafoor et al., 2006). According to Gilbert et al. (2008), in cases where trimester-specific references are not available, an upper limit for TSH in pregnant women may be adopted as $2.5 \mathrm{mIU} / \mathrm{L}$.

Based on the reviewed literature, we argue that the reported ranges of TSH level to be considered as 'normal' vary both spatially and demographically. However, there are consistencies among the study results. A general consensus is that the lower limit of normal to be $0.04 \mathrm{mlU} / \mathrm{L}$ and upper limit of normal to be $2.5 \mathrm{mlU} / \mathrm{L}$ (Negro et al., 2010). For the purpose of present study, we consider 2.5-5.0 mlU/L as the 'high normal', and chose to evaluate the impact of serum TSH levels between 2.5 and $5.0 \mathrm{mIU} / \mathrm{L}$ on first trimester pregnancy loss in anti-TPO Ab negative women. Specifically, we wanted to know the average TSH level during first trimester in anti-TPO Ab negative pregnant women who had miscarriage; the average TSH level during first trimester who did not have miscarriage; and whether there is association between high normal TSH level and first trimester pregnancy loss.

\section{Materials and Methods}

An unmatched case control study involving 119 women in their first trimester was conducted during July 2011 to June 2012 in three tertiary care hospitals in Bangladesh. The study population consisted of in- and out-patients visiting at three facilities in Dhaka namely, Dept. of Obstetrics and Gynaecology under Bangabandhu Sheikh Mujib Medical University (BSMMU), Dhaka Medical College Hospital (DMCH), and Mohammadpur Fertility Services and Training Centre (MFSTC). A purposive sampling scheme was used for data collection.

BRF Www.jBiomedAnalytics.org 
Due to time and financial constraints, we could collect data on 119 subjects for this study, of which 61 were cases and 58 were controls. Because the samples size was small, special attention was paid to keeping the case and control groups as similar as possible. We strictly followed the inclusion and exclusion criteria in order to maintain data quality.

Patients who had spontaneous loss of the fetus (denoted subsequently in this article as miscarriage) during first trimester were in case group while those with continuing pregnancy were in control group. A miscarriage was defined as the spontaneous loss of the fetus. The cases and the controls were further classified into two groups according to the serum TSH level as Group I: TSH level 0.4 to $2.5 \mathrm{mIU} / \mathrm{L}$, and Group II: TSH level $>2.5$ to $5.0 \mathrm{mIU} / \mathrm{L}$. Inclusion criteria for cases include anti-TPO Ab negative women aged 18-45 years; gestational period up to first trimester (12 weeks) who had pregnancy loss diagnosed clinically and confirmed by USG. For the control group, women with the same inclusion criteria of the case group except with continuing pregnancy we included in the study. Patients with the following characteristics were excluded from the study: pregnancy with known thyroid disease; suffering from acute illness-such as viral hepatitis, typhoid, chronic diseases such as chronic hypertension, chronic renal disease, uncontrolled diabetes mellitus; subjects taking medication that interferes with the thyroid function such as steroid, carbamazepine etc. Participants who have lost pregnancy due to any other cause were excluded from the study.

A pre-designed data collection sheet was used to collect data. Informed written consent was taken from each of the study subjects. Ethical clearance for this study was taken from the Institutional Review Board (IRB) of BSMMU. To determine TSH level, $5 \mathrm{ml}$ of venous blood was drawn from ante-cubital vein using disposable syringe with all aseptic precaution. Blood samples were transferred immediately into clean and dry test tubes with gentle push after removal of the needle to avoid hemolysis. The samples were allowed to clot and then centrifuged. Serum was aliquoted into label micro-centrifuged tubes and preserved at 28 degree Celsius for future analysis. Analyses of TPO-Ab and TSH were completed using Abbott Axsym system/AxSYM 3rd Generation TSH assay within five days of sampling.

Data were collected on demographic and socioeconomic variables such as patients' age, height, weight, family income, and husband's occupation. The results in this article were generated using SAS/STAT software, Version 9.4 of the SAS System for Windows. Copyright, SAS Institute Inc. SAS and all other SAS Institute Inc. product or service names are registered trademarks or trademarks of SAS Institute Inc., Cary, NC, USA.

\section{Results and Discussion}

All the study subjects were TPO-Ab negative. On the basis of serum TSH level, study subjects were sub-classified as low normal TSH group (TSH level at or below $2.5 \mathrm{mIU} / \mathrm{L}$ ) and high normal TSH group (TSH level 2.5-5.0 mIU/L). Descriptive statistics such means, medians, and 75th percentiles were calculated and are presented in Table 1. Average age of women in case group was $25 \pm 5.36$ years compared to the age of women in control group which was $24.68 \pm 5.07$. Weight of women in case and control groups were $55.44 \pm 7.98$ and $55.13 \pm 6.95 \mathrm{~kg}$, respectively. Average BMI for subjects in the case group was $25.24 \pm 6.13$ which was similar to the average BMI of $24.98 \pm 4.31$ for the control group subjects. We found no significant difference between cases and controls based on age, weight, and BMI. Thus, we conclude that case and control groups were identical with respect to age, weight, and BMI. 
Table 1: Comparison of respondents in case and control groups by physical and clinical characteristics.

\begin{tabular}{lccc}
\hline & Case $(N=61)$ & Control $(N=58)$ & p-value for $t$-test \\
\hline Age (years) & & & \\
Mean \pm SD & $25.06 \pm 5.36$ & $24.68 \pm 5.07$ & .6910 \\
Median & 25 & 24 & \\
75th percentile & 28 & 28 & \\
& & & \\
Weight (kg) & & & \\
Mean \pm SD & $55.44 \pm 7.58$ & $55.13 \pm 6.95$ & .8087 \\
Median & 54 & 56 & \\
75th percentile & 60 & 90 & \\
& & & \\
BMI & & & \\
Mean \pm SD & $25.24 \pm 6.13$ & $24.98 \pm 4.31$ & .7848 \\
Median & 23.68 & 24.19 & \\
75th percentile & 25.82 & 26.73 & \\
& & & \\
Serum TSH (mlU/L) & & & \\
Mean \pm SD & $1.74 \pm 1.15$ & $1.42 \pm 0.86$ & \\
Median & 1.45 & 1.15 & \\
75th percentile & 2.16 & 1.92 & \\
\hline
\end{tabular}

The average serum TSH level was $1.74 \mathrm{mlU} / \mathrm{L}$ in the case group, and $1.42 \mathrm{mlU} / \mathrm{L}$ in the control group. The median TSH levels were $1.45 \mathrm{mlU} / \mathrm{L}$ and $1.15 \mathrm{mlU} / \mathrm{L}$ for case and control groups, respectively. The 75th percentile for the TSH level was 2.16 for case group and 1.92 for control group. The percentiles indicate that $25 \%$ of the subjects in the case group had serum TSH levels above $2.16 \mathrm{mlU} / \mathrm{L}$ while $25 \%$ of the control subjects had TSH level above $1.92 \mathrm{mlU} / \mathrm{L}$. These results show evidence that case group had much higher serum TSH level than the control group. However, the difference was not statistically significant $(\mathrm{p}=.0841)$ at $5 \%$ level of significance.

In order to know if the serum TSH level was associated with first trimester pregnancy loss, we constructed a $2 \times 2$ table where TSH level and the exposure status (case vs control) was cross-classified (Table 2). We had nearly equal number of cases and control patients whose serum TSH levels were at or below $2.5 \mathrm{mlU} / \mathrm{L}$ (49 in case group vs 52 in control group). On the other hand, there were twice as many subjects in the case group (12) whose TSH levels were greater than $2.5 \mathrm{mlU} / \mathrm{L}$ compared to 6 subjects in the control group. Among the subjects with high normal TSH, $66.67 \%$ were cases compared to $33.33 \%$ controls. As such, there were $33 \%$ more women with high normal TSH level ( $>2.5 \mathrm{mIU} / \mathrm{L})$ who lost pregnancy than those women who had continuing pregnancy. However, we did not find statistically significant association between first trimester pregnancy loss and high normal TSH level (Pearson Chi-square $=2.01, p=.1558$ ). This indicates that case and control groups do not differ significantly with respect to high normal serum TSH levels.

?RF WWw.jBiomedAnalytics.org 
Table 2: Cross-tabulation of TSH level and exposure to event (miscarriage).

\begin{tabular}{lccc}
\hline & Case & Control & Chi-square (p-value) \\
\hline Group I (low normal) & 49 & 52 & \\
TSH $\leq 2.5 \mathrm{ml} \mathrm{U} / \mathrm{L}$ & & & $2.01(.1558)$ \\
& & & \\
Group II (high normal) & 12 & 6 & \\
TSH $>2.5 \mathrm{mlU} / \mathrm{L}$ & & & \\
\hline
\end{tabular}

\section{Conclusion}

The aim of this study was to know the average serum TSH levels for women who had miscarriage, and those who did not have miscarriage during the first trimester of pregnancy. We found average TSH level for the control group as $1.42 \pm 0.86 \mathrm{mlU} / \mathrm{L}$, which was lower than the average TSH level for the case group (1.74 $\pm 1.15 \mathrm{mlU} / \mathrm{L})$. However, no statistically significant association was found between the serum TSH level and first trimester pregnancy loss among anti-TPO Ab negative women. Our observations are in agreement with that of Stagnaro-Green et al. (1990) but quite different from the findings of Negro et al. (2010).

To the best of our knowledge, this was the first such study to understand the association between TSH level and first trimester pregnancy loss among anti-TPO antibody negative women in Bangladesh. It is to be noted that the findings are based on data collected from three tertiary care hospitals only. As such, the results should be interpreted with caution. However, findings of this study will provide a baseline for any future study related to this topic involving Bangladeshi women. In addition, our results contribute to the growing discussion on redefining the lower limit for the 'high normal' serum TSH level among the anti-TPO Ab negative women.

There were a number of limitations of this study. First, the sample size was not adequate for the case and control groups, which may have resulted in loss of power to detect significant association between TSH level and first trimester pregnancy loss. Second, there could be spatial variation among the study subjects which may have affected the results. Therefore, large-scale multi-centered studies would be necessary to capture spatial variation as well as to have higher power for the statistical tests to detect significant differences.

\section{Declarations}

\section{Acknowledgments}

The first author is grateful to Bangabandhu Sheikh Mujib Medical University for supporting the conduct of the research study.

\section{Funding}

None 


\section{Conflict of Interest}

Authors have declared no conflict of interest.

\section{Ethical Approval}

Ethical clearance for this study was taken from the Institutional Review Board (IRB) of Bangabandhu Sheikh Mujib Medical University (BSMMU).

\section{References}

Casey BM, Leveno KJ (2006). "Thyroid disease in pregnancy." Obstetrics \& Gynecology, 108(5), 1283-1292. doi:10.1097/01.AOG.0000244103.91597.c5.

De Groot L, Abalovich M, Alexander EK, Amino N, Barbour L, Cobin RH, Eastman CJ, Lazarus $\mathrm{JH}$, Luton D, Mandel SJ, et al. (2012). "Management of thyroid dysfunction during pregnancy and postpartum: an Endocrine Society clinical practice guideline." The Journal of Clinical Endocrinology \& Metabolism, 97(8), 2543-2565. doi:10.1210/jc.2011-2803.

Ghafoor F, Mansoor M, Malik T, Malik MS, Khan AU, Edwards R, Akhtar W (2006). "Role of thyroid peroxidase antibodies in the outcome of pregnancy." Journal of the College of Physicians and Surgeons-Pakistan: JCPSP, 16(7), 468-471. doi:7.2006/JCPSP.468471.

Gilbert RM, Hadlow NC, Walsh JP, Fletcher SJ, Brown SJ, Stuckey BG, Lim EM (2008). "Assessment of thyroid function during pregnancy: first-trimester (weeks 9-13) reference intervals derived from Western Australian women." The Medical Journal of Australia, 189(5), 250-253.

Negro R, Schwartz A, Gismondi R, Tinelli A, Mangieri T, Stagnaro-Green A (2010). "Increased pregnancy loss rate in thyroid antibody negative women with TSH levels between 2.5 and 5.0 in the first trimester of pregnancy." The Journal of Clinical Endocrinology \& Metabolism, 95(9), E44-E48. doi:10.1210/jc.2010-0340.

Panesar N, Li C, Rogers M (2001). "Reference intervals for thyroid hormones in pregnant Chinese women." Annals of clinical biochemistry, 38(4), 329-332. doi:10.1258/0004563011900830.

Pearce E, Oken E, Gillman M, Lee S, Magnani B, Platek D, Braverman L (2008). "Association of first-trimester thyroid function test values with thyroperoxidase antibody status, smoking, and multivitamin use." Endocrine Practice, 14(1), 33-39. doi:10.4158/EP.14.1.33.

Quinn FA, Gridasov GN, Vdovenko SA, Krasnova NA, Vodopianova NV, Epiphanova MA, Schulten M (2005). "Prevalence of abnormal thyroid stimulating hormone and thyroid peroxidase antibody-positive results in a population of pregnant women in the Samara region of the Russian Federation." Clinical Chemical Laboratory Medicine, 43(11), 12231226. doi:10.1515/CCLM.2005.212.

Stagnaro-Green A, Roman SH, Cobin RH, El-Harazy E, Alvarez-Marfany M, Davies TF (1990). "Detection of at-risk pregnancy by means of highly sensitive assays for thyroid autoantibodies." Jama, 264(11), 1422-1425. doi:10.1097/00006254-199103000-00005.

BRF Www.jBiomedAnalytics.org 
Stricker R, Echenard M, Eberhart R, Chevailler M, Perez V, Quinn F, Stricker R (2007). "Evaluation of maternal thyroid function during pregnancy: the importance of using gestational age-specific reference intervals." European Journal of Endocrinology, 157(4), 509-514. 